\section{Künstliches Koma als Qualitätsmarker}

Das „künstliche Koma“ ist für den Neuromediziner ebenfalls ein rotes Tuch. „Ein Unfall kann gar nicht schwer gewesen sein, wenn der Patienten nicht in ein künstliches Koma versetzt wird. Dass es Hirnverletzungen gibt, die per se zum Koma führen, wird oft ausgelassen. "Der Begriff werde inflationär benutzt, und dazu würden auch Ärzte beitragen, die das künstliche Koma zunehmend als eine Art Qualitätsmarker für die Behandlung verstünden. „Stellen Sie sich vor, der Arzt würde gefragt, ob der prominente Patient jetzt im künstlichen Koma sei, und man würde der Presse sagen: Nein, der ist sowieso schon komatös." Vermutlich würde die sich dann einen anderen Experten suchen. „In Deutschland findet man für jedes noch so abwegige Thema einen ,Fachmann', der sich aus guten, meist finanziellen Gründen mit diesem Thema beschäftigt und das zum Besten gibt, was das Publikum hören möchte." Wer mit solchen Personen als wissenschaftlich denkender Mensch konfrontiert werde, habe wenig zu lachen: „Gegen die Phalanx aus Leid, Betroffenheit und tenden- ziöser Berichterstattung hat man keine Chance“, so Hacke. Wenig hilfreich sei es auch, wenn „Parkinson-Leute über Schlaganfall und MS-Größen über Intensivmedizin reden, nur weil die Presse zufällig auf deren Namen gestoßen ist. Wir scheuen uns viel zu häufig, bei Anfragen zu sagen, dass wir einen anderen Spezialisten empfehlen würden." Hacke forderte daher eine schlagkräftige, manchmal auch kontroverse Pressearbeit und ein gutes Medientraining. „Wir brauchen medienerfahrene Spezialisten für die verschiedenen Themenbereiche der Neurointensivmedizin, die bei Anfragen dann auch vorgeschlagen werden.“ Sonst würden Themen aus der Neurointensivmedizin weiterhin von Angehörigen anderer Disziplinen präsentiert oder von Neurologen, die eine Intensivstation schon seit Jahren nicht mehr von innen gesehen haben. „Und so sind dann auch die Beiträge.“

Thomas Müller, Springer Medizin

Präsidentensymposium. Vortrag Professor Werner Hacke: Neurointensivmedizin in der öffentlichen Wahrnehmung. 33. ANIM, Berlin, 28.-30.1.2016

\title{
Nach Schlaganfall nicht zu früh bewegen!
}

\author{
Patienten nach Schlaganfall sollten nicht zu früh \\ mobilisiert und stimuliert werden - dies könnte den \\ Zelluntergang in der Infarktrandzone verstärken.
}

Eine gute Reha kann bei vielen Schlaganfallpatienten die Mobilität erhöhen. Das weckt die Hoffnung, eine möglichst frühe Bewegung und Stimulation - vielleicht noch in der Akutphase könnte einen Teil der sensomotorischen Defizite verhindern. In Tierversuchen scheint dies möglich zu sein, allerdings nur in bestimmten Phasen - danach schadet die Stimulation eher, berichtete Professor Matthias Endres, Charité Berlin. So gelang es etwa bei Mäusen, die Folgen eines künstlich erzeugten ischämischen Schlaganfalls deutlich zu minimieren oder den Zelluntergang ganz zu verhindern, wenn die entsprechenden Areale um den Infarktzeitraum herum - am besten noch vor dem Infarkt - sensomotorisch aktiviert wurden, etwa durch Bewegung der Vibrissen. Durch die neurovaskuläre Koppelung würden verstärkt Kollateralen im Infarktgebiet aktiviert, die für eine verbesserte Perfusion sorgten, so die Erklärung für das Phänomen.

\section{Patienten "wie ein rohes Ei behandeln"}

Hat sich jedoch bereits ein Infarktkern gebildet, werden gegenläufig wirkende Mechanismen beobachtet, sagte Endres. Dann bildet sich um das Infarktareal eine metastabile Zone. Forscher konnten durch Ableitungen in Tierversuchen zahlreiche Depolarisationen in der Randzone nachweisen. Offenbar versucht das gefährdete Gewebe hier, Ionengradienten wiederherzustellen oder aufrechtzuerhalten. Das ist offenbar mit einem erhöhten Energieverbrauch verbunden. Stimulierten die Forscher nun gezielt Vorderpfotenbereiche, welche die Infarktrandzonen beanspruchen, nahmen solche Periinfarkt-Depolarisationen zu, sagte Endres. Zugleich vergrößerte sich die Infarktzone und das funktionelle Ergebnis wurde schlechter. Die Erklärung hierfür: Eine Stimulation steigert in den metastabilen Randzonen den
Energiebedarf - nicht gerade günstig, wenn das Gewebe ums Überleben kämpft. Einen ähnlichen Effekt sehen Forscher auch, wenn es zu einem Blutdruckabfall oder Sauerstoffmangel kommt. Übertragen auf den Menschen könnten solche Mechanismen durchaus relevant sein, da sehr niedrige Blutdrücke nicht selten auch bei Schlaganfallpatienten in der Akutphase beobachtet werden, sagte Endres. Und was die frühe Stimulation betrifft: „Wir müssten Schlaganfallpatienten nach diesen Ergebnissen in der Akutphase also eher wie ein rohes Ei behandeln, um sie besser vor einem erhöhten Energiebedarf zu schützen."

\section{SSRI schützen dopaminerge Neuronen}

Auf eine andere Option, den Schaden zu begrenzen, weisen ebenfalls Experimente an Mäusen, nach denen SSRI bei ihnen apathieähnliche Zustände nach einem Schlaganfall verhindern. So zeigt das MRT bei Mäusen etwa vier Tage nach dem Infarkt ein ödemartiges Signal im Bereich der ipsilateralen Substantia nigra, unter SSRI blieben die Auffälligkeiten weitgehend aus. Bei Menschen lassen sich ähnliche Signale etwa sechs bis zehn Tage nach ischämischem Infarkt nachweisen. Endres geht davon aus, dass SSRI den sekundären exofokalen Zelluntergang bremsen. Immerhin gibt es erste Hinweise, dass dies auch bei Menschen klappt. In der FLAME-Studie [Chollet F et al. Lancet Neurol 2011; 10: 123 - 30] bekamen jeweils knapp 60 Patienten fünf bis zehn Tage nach einem Schlaganfall für drei Monate Fluoxetin oder Placebo. Unter dem SSRI traten nicht nur Depressionen viel seltener auf (7\% vs. $29 \%$ ), auch das funktionelle Ergebnis nach drei Monaten war deutlich besser: Einen mRS-Wert von 0-2 Punkten erreichten $34 \%$ mit Fluoxetin, aber nur $11 \%$ mit Placebo. „Das ist ein schönes Beispiel für die gute Korrelation von präklinischen Experimenten und klinischen Studien“, so Endres.

Thomas Müller, Springer Medizin

Symposium der Deutschen Schlaganfallgesellschaft (DSG), Vortrag Professor Matthias Endres: Neue Therapieoptionen. 33. ANIM, Berlin 28. -30.1.2016 